\title{
On the Secondary Resonance of a Spinning Disk Under Space-Fixed Excitations
}

Jen-San Chen

Professor

Chin-Yi Hua

Graduate Student

\section{Chia-Min Sun}

Graduate Student

Department of Mechanical Engineering, National Taiwan University, Taipei, Taiwan 10617
We investigate the possibility of secondary resonance of a spinning disk under space-fixed excitations. Von Karman's plate model is employed in formulating the equations of motion of the spinning disk. Galerkin's procedure is used to discretize the equations of motion, and the multiple scale method is used to predict the steady state solutions. Attention is focused on the nonlinear coupling between a pair of forward (with frequency $\omega_{m \bar{n}}$ ) and backward (with frequency $\omega_{m n}$ ) traveling waves. It is found that combination resonance may occur when the excitation frequency is close to $2 \omega_{m n}+\omega_{m \bar{n}}, \omega_{m n}+2 \omega_{m \bar{n}}$, or $1 / 2\left(\omega_{m \bar{n}}+\omega_{m n}\right)$. When the combination resonance does occur, the frequencies of the free oscillation components are shifted slightly from the respective natural frequencies $\omega_{m \bar{n}}$ and $\omega_{m n}$. The final response is therefore quasiperiodic. However, in the case when the excitation frequency is close to $1 / 2\left(\omega_{m \bar{n}}-\omega_{m n}\right)$, no combination resonance is possible. In the case when the excitation frequency is close to $1 / 3 \omega_{m n}$ and $1 / 2\left(\omega_{m \bar{n}}-\omega_{m n}\right)$ simultaneously, internal resonance between the forward and backward modes can occur. The frequencies of the free oscillation components are exactly three times and five times that of the excitation frequency. In this special case both saddle-node and Hopf bifurcations are observed. [DOI: 10.1115/1.1760562]

\section{Introduction}

The dynamic response of a spinning flexible disk under spacefixed excitation has been under intensive research in the past decades, partly due to its potential applications in many mechanical design problems such as circular saws and modern disk drives. Benson and Bogy [1] and Cole and Benson [2] investigated the steady state response of a spinning disk under a space-fixed point force. Ono and Maeno [3] and Jiang et al. [4] studied the response of a spinning floppy disk under space-fixed harmonic excitation. Chen and Hsu $[5,6]$ considered the transient response as well as steady state deflections of a spinning disk under space-fixed stepforces and couples.

These aforementioned analyses all used linearized plate equation. In some cases when the disk deflection becomes excessive the stiffening effect due to membrane stretching has to be taken into account. This nonlinear stretching effect is commonly treated by adopting von Karman's plate model [7]. Tobias and Arnold [8] studied the influence of imperfection on the nonlinear vibration of rotating disks. Maher and Adams [9] calculated the steady state point-load deflection of a disk spinning near a base plate. Torii et al. [10] investigated analytically and experimentally the primary resonance of a spinning disk under both rotating and spacefixed loads simultaneously. Raman and Mote [11] and Chen [12] studied the nonlinear oscillation of a disk spinning near its critical speed. These researches mainly dealt with the primary resonance of a spinning disk, i.e., the excitation frequency is close to one of the natural frequencies of the spinning disk. On the other hand, the secondary resonance of a spinning disk under space-fixed excitation has not been studied in the literature. When the disk is not spinning, however, subharmonic resonance was reported by Nayfeh and Vakakis [13].

In this paper we investigate the possibility of secondary resonance of a spinning disk under space-fixed excitations. The equations of motion are formulated by using von Karman's plate model. Galerkin's method is then employed to discretize the equations of motion. Attention is focused on the nonlinear coupling

Contributed by the Technical Committee on Vibration and Sound for publication in the JOURNAL OF VIBRATION AND ACOUSTICS. Manuscript received June 2001; Revised Dec. 2003. Associate Editor: J. P. Cusumano. between a pair of forward and backward traveling waves. The steady state solutions are predicted by multiple scale method and verified by Runge-Kutta integration.

\section{Equations of Motion}

We consider an elastic circular disk spinning with constant speed $\Omega$. The equations of motion of the spinning disk in terms of transverse displacement $w$ and stress function $\phi$ can be written as [7]

$$
\begin{gathered}
\rho h\left(w_{, t t}+2 \Omega w_{, t \theta}+\Omega^{2} w_{, \theta \theta}\right)+c_{f} w_{, t}+D \nabla^{4} w \\
=h\left\{w_{, r r}\left(r^{-1} \phi_{, r}+r^{-2} \phi_{, \theta \theta}\right)+\left(r^{-1} w_{, r}+r^{-2} w_{, \theta \theta}\right) \phi_{, r r}\right. \\
\left.-2\left(r^{-1} w_{, \theta}\right)_{, r}\left(r^{-1} \phi_{, \theta}\right)_{, r}-\rho \Omega^{2} r\left(\frac{r}{2} \nabla^{2} w+w_{, r}\right)\right\} \\
+q(r, \theta) \cos \gamma t \\
\nabla^{4} \phi=-E\left[w_{, r r}\left(r^{-1} w_{, r}+r^{-2} w_{, \theta \theta}\right)+2 r^{-3} w_{, r \theta} w_{, \theta}-r^{-2}\left(w_{, r \theta}\right)^{2}\right. \\
\left.-r^{-4}\left(w_{, \theta}\right)^{2}\right]+2(1-\nu) \rho \Omega^{2}
\end{gathered}
$$

$(r, \theta)$ are space-fixed polar coordinates. The parameters $\rho, h, E, \nu$, and $D$ are the mass density, thickness, Young's modulus, Poisson ratio, and flexural rigidity of the disk, respectively. $c_{f}$ represents a space-fixed homogeneous damping due to the surrounding air. $q(r, \theta) \cos \gamma t$ is the space-fixed harmonic loading. In writing Eq. (2) the in-plane inertia is neglected. The disk is assumed to be "partially" clamped [1] at the inner radius $r=a$ and is free at the outer radius $r=b$.

It is noted that while Eqs. (1) and (2) are nonlinear in terms of $w$, they are linear in $\phi$. Therefore we can divide the stress function $\phi$ in Eq. (2) into homogeneous solution $\phi_{1}$ and particular solution $\phi_{2}$. $\phi_{1}$ accounts for the stretching effect due to the centrifugal force, and $\phi_{2}$ involves nonlinear terms of $w$. After substituting the relation $\phi=\phi_{1}+\phi_{2}$ in Eqs. (1) and (2) and introducing the following dimensionless quantities, 


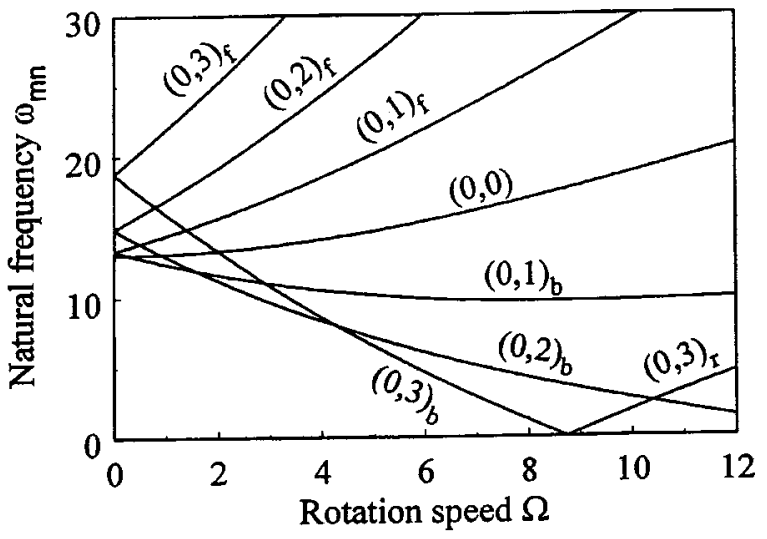

Fig. 1 Natural frequency loci of a freely spinning disk

$$
\begin{gathered}
t^{*}=\frac{t}{b^{2}} \sqrt{\frac{D}{\rho h}}, \quad \Omega^{*}=\Omega b^{2} \sqrt{\frac{\rho h}{D}}, \quad \gamma^{*}=\gamma b^{2} \sqrt{\frac{\rho h}{D}}, \\
r^{*}=\frac{r}{b}, \quad w^{*}=w \sqrt{\frac{b}{h^{3}}}, \\
\phi^{*}=\phi \frac{h}{D}, \quad c_{f}^{*}=c_{f} \frac{b^{3}}{24\left(1-\nu^{2}\right) \sqrt{\rho h^{3} D}}, \quad \varepsilon=12\left(1-\nu^{2}\right) \frac{h}{b}, \\
\eta=\frac{a}{b}, \\
\sigma_{r}^{*}=\frac{h b^{2}}{D} \sigma_{r}, \quad q^{*}=q \sqrt{\frac{b^{9}}{D^{2} h^{3}}},
\end{gathered}
$$

we can rewrite the equations of motion in the dimensionless form after dropping the asterisks for simplicity,

$$
\begin{aligned}
w_{, t t}+ & 2 \Omega w_{, t \theta}+\Omega^{2} w_{, \theta \theta}+2 \varepsilon c_{f} w_{, t}+\nabla^{4} w-r^{-1}\left(\sigma_{r} r w_{, r}\right)_{, r} \\
& -r^{-2} \sigma_{\theta} w_{, \theta \theta} \\
= & w_{, r r}\left(r^{-1} \phi_{2, r}+r^{-2} \phi_{2, \theta \theta}\right)+\left(r^{-1} w_{, r}+r^{-2} w_{, \theta \theta}\right) \phi_{2, r r} \\
& -2\left(r^{-1} w_{, \theta}\right)_{, r}\left(r^{-1} \phi_{2, \theta}\right)_{, r}+q(r, \theta) \cos \gamma t \\
\nabla^{4} \phi_{2}= & -\varepsilon\left[w_{, r r}\left(r^{-1} w_{, r}+r^{-2} w_{, \theta \theta}\right)+2 r^{-3} w_{, r \theta} w_{, \theta}-r^{-2}\left(w_{, r \theta}\right)^{2}\right. \\
& \left.-r^{-4}\left(w_{, \theta}\right)^{2}\right]
\end{aligned}
$$

where $\sigma_{r}$ and $\sigma_{\theta}$ are due to centrifugal force,

$$
\begin{gathered}
\sigma_{r}=\frac{3+\nu}{8} \Omega^{2}\left(1-r^{2}\right) \\
\sigma_{\theta}=\frac{1}{8} \Omega^{2}\left[(3+\nu)-(1+3 \nu) r^{2}\right]
\end{gathered}
$$

In the special case when $\varepsilon=0$, the solution $\phi_{2}$ in Eq. (4) is identically zero, and as a consequence Eq. (3) for an undamped freely spinning disk is reduced to

$$
w_{, t t}+2 \Omega w_{, t \theta}+\Omega^{2} w_{, \theta \theta}+\nabla^{4} w-r^{-1}\left(\sigma_{r} r w_{, r}\right)_{, r}-r^{-2} \sigma_{\theta} w_{, \theta \theta}=0
$$

The natural frequency of a mode with $n$ nodal diameters and $m$ nodal circles is denoted by $\omega_{m n}$. The corresponding eigenfunction is complex and assumes the form

$$
w_{m n}(r, \theta)=R_{m n}(r) e^{i n \theta}
$$

It is also noted that the eigenfunctions $w_{m n}$ are orthonormal.

Figure 1 shows the natural frequency loci of a spinning disk as the rotation speed varies. The clamping ratio $\eta$ is 0.5 , and the Poisson ratio $\nu$ is 0.27 . Only the modes with less than four nodal diameters are shown here. The subscripts $f, b$, and $r$ of the mode label $(m, n)$ represent forward, backward, and reflected modes, respectively [6].

\section{Discretization}

In this paper we focus on the secondary resonance of a pair of forward and backward $(m, n)$ modes excited by the space-fixed load and assume that the influence of other modes on this specific pair is negligible. The solution $w(r, \theta, t)$ of Eqs. (3) and (4) can then be approximated in terms of eigenfunctions $w_{m n}(r, \theta)$ as

$$
w(r, \theta, t)=c_{m n}(t) w_{m n}+\bar{c}_{m n}(t) \bar{w}_{m n}
$$

Both $c_{m n}(t)$ and $w_{m n}(r, \theta)$ in Eq. (9) are complex functions, while the displacement $w(r, \theta, t)$ is a real function. $\bar{w}_{m n}$ represents the complex conjugate of $w_{m n}$. In order to solve $\phi_{2}$ in Eq. (4) we introduce a set of eigenfunctions $\phi_{m n}$ satisfying the following differential equation,

$$
\nabla^{4} \phi_{m n}-\beta_{m n}^{4} \phi_{m n}=0
$$

$\phi_{m n}$ satisfy the same boundary conditions as $\phi_{2}$ does. After expressing $\phi_{2}$ in terms of eigenfunction series $\phi_{m n}$ and following Galerkin's procedure, we can discretize Eqs. (3) and (4) into

$$
\ddot{c}_{m n}+2 i n \Omega \dot{c}_{m n}+\kappa_{m n} c_{m n}+2 \varepsilon c_{f} \dot{c}_{m n}+\varepsilon \alpha\left|c_{m n}\right|^{2} c_{m n}=q_{m n} \cos \gamma t
$$

where

$$
\begin{aligned}
& \kappa_{m n}=\omega_{m n} \omega_{m \bar{n}} \\
& q_{m n}=\int_{\theta=0}^{2 \pi} \int_{r=\eta}^{1} q(r, \theta) R_{m n}(r) e^{-i n \theta} r d r d \theta
\end{aligned}
$$

Equation (11) represents a gyroscopic system with two degrees of freedom. $\omega_{m n}$ and $\omega_{m \bar{n}}$ are the natural frequencies of the backward and the forward modes, respectively. It is noted that $\omega_{m \bar{n}}$ is equal to $\omega_{m n}+2 n \Omega$. The excitation frequency $\gamma$ is assumed to be away from both $\omega_{m n}$ and $\omega_{m \bar{n}}$. In other words, we exclude the case of primary resonance. The thickness parameter $\varepsilon$ is assumed to be small. It is noted that the damping and the nonlinear terms are of order $\varepsilon$, while the forcing term is of order one. Without loss of generality the load parameter $q_{m n}$ can be taken as real. Constant $\alpha$ can be obtained via numerical integration involving eigenfunctions $w_{m n}$ and $\phi_{m n} \cdot\left|c_{m n}\right|$ represents the absolute value of complex number $c_{m n}$.

\section{Multiple Scale Method}

In order to find the steady state oscillation we apply the method of multiple scale [14] to analyze Eq. (11). The method of multiple scale assumes an expansion of the solution in the form

$$
c_{m n}(t)=c_{m n}^{(0)}\left(t, T_{1}\right)+\varepsilon c_{m n}^{(1)}\left(t, T_{1}\right)+O\left(\varepsilon^{2}\right)
$$

where $T_{1} \equiv \varepsilon t$. Substituting (14) into (11) and equating coefficients of like powers of $\varepsilon$ yields

$$
\begin{array}{cl}
\varepsilon^{0}: & D_{0}^{2} c_{m n}^{(0)}+2 i n \Omega D_{0} c_{m n}^{(0)}+\kappa_{m n} c_{m n}^{(0)}=q_{m n} \cos \gamma t \\
\varepsilon^{1}: & D_{0}^{2} c_{m n}^{(1)}+2 i n \Omega D_{0} c_{m n}^{(1)}+\kappa_{m n} c_{m n}^{(1)}=-2 D_{1} D_{0} c_{m n}^{(0)} \\
& -2 i n \Omega D_{1} c_{m n}^{(0)}-2 c_{f} D_{0} c_{m n}^{(0)}-\alpha\left|c_{m n}^{(0)}\right|^{2} c_{m n}^{(0)}
\end{array}
$$

where $D_{0} \equiv \partial / \partial t$, and $D_{1} \equiv \partial / \partial T_{1}$. The general solution of Eq. (15) can be written in the form

$$
c_{m n}^{(0)}=d_{1}\left(T_{1}\right) e^{i \omega_{m n} T_{0}}+d_{2}\left(T_{1}\right) e^{-i \omega_{m \bar{n}} T_{0}}+\Lambda_{1} e^{i \gamma T_{0}}+\Lambda_{2} e^{-i \gamma T_{0}}
$$

where 
Table 1 Six cases in which secular terms may occur in the solution $c_{m n}^{(1)}$ in Eq. (14)

\begin{tabular}{cc}
\hline \hline Case & Closeness condition \\
\hline 1 & $\gamma \approx \frac{1}{3} \omega_{m \bar{n}}$ \\
2 & $\gamma \approx \frac{1}{3} \omega_{m n}$ \\
3 & $\gamma \approx 2 \omega_{m n}+\omega_{m \bar{n}}$ \\
4 & $\gamma \approx \omega_{m n}+2 \omega_{m \bar{n}}$ \\
5 & $\gamma \approx \frac{1}{2}\left(\omega_{m \bar{n}}+\omega_{m n}\right)$ \\
6 & $\gamma \approx \frac{1}{2}\left(\omega_{m \bar{n}}-\omega_{m n}\right)$ \\
\hline
\end{tabular}

$$
\begin{aligned}
& \Lambda_{1}=\frac{q_{m n}}{2\left(-\gamma^{2}-2 n \Omega \gamma+\kappa_{m n}\right)} \\
& \Lambda_{2}=\frac{q_{m n}}{2\left(-\gamma^{2}+2 n \Omega \gamma+\kappa_{m n}\right)}
\end{aligned}
$$

Substituting Eq. (17) into the right hand side of Eq. (16) we observe that there are six cases in which secular terms may occur in the solution $c_{m n}^{(1)}$. These six cases are listed in Table 1. Further analysis will reveal that there are three possible outcomes: (A) Single-mode resonance: In cases (1) and (2) only a single mode will be excited in the superharmonic manner. (B) Combination resonance: In cases (3) to (6) the secondary resonance involves both the forward and backward modes. (C) Simultaneous resonance: In the event when cases (2) and (6) occur simultaneously, in other words $\omega_{m \bar{n}}$ is close to $5 / 3 \omega_{m n}$ and $\gamma$ is close to $1 / 3 \omega_{m n}$, then internal resonance involving both modes will occur. In the following we will discuss these three possible outcomes one by one.

\section{Single-Mode Superharmonic Resonance}

We first consider the case when the excitation frequency is close to $1 / 3 \omega_{m \bar{n}}$. We define the frequency detuning parameter $s$ by

$$
\gamma=\frac{1}{3} \omega_{m \bar{n}}+\varepsilon \varsigma
$$

The secular terms of Eq. (16) can be eliminated if

$$
\begin{aligned}
& 2 i \hat{\omega}_{m n} D_{1} d_{1}+i 2 \omega_{m n} c_{f} d_{1}+\alpha d_{1}\left(\left|d_{1}\right|^{2}+2\left|d_{2}\right|^{2}+2 \Lambda_{1}^{2}+2 \Lambda_{2}^{2}\right)+G_{1} \\
& \quad=0 \\
& 2 i \hat{\omega}_{m n} D_{1} d_{2}+i 2 \omega_{m \bar{n}} c_{f} d_{2}-\alpha d_{2}\left(\left|d_{2}\right|^{2}+2\left|d_{1}\right|^{2}+2 \Lambda_{1}^{2}+2 \Lambda_{2}^{2}\right)+G_{2} \\
& \quad=0
\end{aligned}
$$

where $\hat{\omega}_{m n}=1 / 2\left(\omega_{m n}+\omega_{m \bar{n}}\right)$. The terms $G_{1}$ and $G_{2}$ are introduced because of the closeness relation Eq. (20),

$$
\begin{gathered}
G_{1}=0 \\
G_{2}=-\alpha \Lambda_{1} \Lambda_{2}^{2} e^{-i 3 \varsigma T_{1}}
\end{gathered}
$$

We express $d_{1}$ and $d_{2}$ in the forms

$$
\begin{aligned}
& d_{1}\left(T_{1}\right)=\frac{1}{2} a_{1}\left(T_{1}\right) e^{i \beta_{1}\left(T_{1}\right)} \\
& d_{2}\left(T_{1}\right)=\frac{1}{2} a_{2}\left(T_{1}\right) e^{i \beta_{2}\left(T_{1}\right)}
\end{aligned}
$$

After substituting Eqs. (25) and (26) into Eqs. (21) and (22) and separating the real and imaginary parts, we obtain

$$
\begin{gathered}
\hat{\omega}_{m n} a_{1}^{\prime}=-c_{f} \omega_{m n} a_{1}+H_{1} \\
8 a_{1} \hat{\omega}_{m n} \beta_{1}^{\prime}=\alpha a_{1}\left(a_{1}^{2}+2 a_{2}^{2}+8 \Lambda_{1}^{2}+8 \Lambda_{2}^{2}\right)+H_{2} \\
\hat{\omega}_{m n} a_{2}^{\prime}=-c_{f} \omega_{m \bar{n}} a_{2}+H_{3} \\
8 a_{2} \hat{\omega}_{m n} \beta_{2}^{\prime}=-\alpha a_{2}\left(a_{1}^{2}+2 a_{2}^{2}+8 \Lambda_{1}^{2}+8 \Lambda_{2}^{2}\right)+H_{4}
\end{gathered}
$$

where the superposed prime denotes differentiation with respect to $T_{1}$. The terms $H_{1}, H_{2}, H_{3}$, and $H_{4}$ are again introduced by the closeness relation Eq. (20),

$$
\begin{gathered}
H_{1}=H_{2}=0 \\
H_{3}=-\alpha \Lambda_{1} \Lambda_{2}^{2} \sin \psi \\
H_{4}=-8 \alpha \Lambda_{1} \Lambda_{2}^{2} \cos \psi
\end{gathered}
$$

where

$$
\psi=3 \varsigma T_{1}+\beta_{2}
$$

We can conclude from Eq. (27) that the steady state solution of $a_{1}$ is always zero. On the other hand the steady state solutions of $a_{2}$ and $\psi$ satisfy the following conditions

$$
\begin{gathered}
c_{f} \omega_{m \bar{n}} a_{2}+\alpha \Lambda_{1} \Lambda_{2}^{2} \sin \psi=0 \\
-\alpha a_{2}\left(a_{2}^{2}+8 \Lambda_{1}^{2}+8 \Lambda_{2}^{2}\right)-8 \alpha \Lambda_{1} \Lambda_{2}^{2} \cos \psi+24 \hat{\omega}_{m n} \varsigma a_{2}=0
\end{gathered}
$$

After eliminating $\psi$ from Eqs. (35) and (36), we obtain the relation between $a_{2}$ and $\mathrm{s}$ as,

$$
\varsigma=\frac{\alpha}{3 \hat{\omega}_{m n}}\left(\frac{1}{8} a_{2}^{2}+\Lambda_{1}^{2}+\Lambda_{2}^{2}\right) \pm \frac{1}{3 \hat{\omega}_{m n}}\left(\frac{\alpha^{2} \Lambda_{1}^{2} \Lambda_{2}^{4}}{a_{2}^{2}}-c_{f}^{2} \omega_{m \bar{n}}^{2}\right)^{1 / 2}
$$

The steady state vibration of the spinning disk is then

$$
\begin{aligned}
w(r, \theta, t)= & R_{m n}(r)\left[a_{2} \cos (-3 \gamma t+\psi+n \theta)+2 \Lambda_{1} \cos (\gamma t+n \theta)\right. \\
& \left.+2 \Lambda_{2} \cos (-\gamma t+n \theta)\right]+O(\varepsilon)
\end{aligned}
$$

It is noted that the frequency of free oscillation component is tuned exactly to $3 \gamma$ by the nonlinearity. Therefore, the final response Eq. (38) is periodic.

The stability of the steady state solutions can be analyzed by expressing $d_{1}\left(T_{1}\right)$ and $d_{2}\left(T_{1}\right)$ as

$$
\begin{aligned}
& d_{1}\left(T_{1}\right)=d_{1}^{(s)}+\hat{d}_{1}\left(T_{1}\right) \\
& d_{2}\left(T_{1}\right)=d_{2}^{(s)}+\hat{d}_{2}\left(T_{1}\right)
\end{aligned}
$$

$d_{1}^{(s)}$ and $d_{2}^{(s)}$ are the steady state solutions. After substituting Eqs. (39) and (40) into Eqs. (21) and (22) and linearizing with respect to the variations $\hat{d}_{1}\left(T_{1}\right)$ and $\hat{d}_{2}\left(T_{1}\right)$ we can obtain a Jacobian matrix. By solving the eigenvalues of the Jacobian matrix we can determine the stability of the steady state solutions. For unstable solutions there exists at least one eigenvalue with positive real part. It is noted that the results for the case of single-mode superharmonic resonances are similar to the results of a single degreeof-freedom Duffing oscillator [14]. Similar single-mode superharmonic resonance will also occur when $\gamma \approx 1 / 3 \omega_{m n}$.

\section{Combination Resonance}

For combination resonance we first consider case (3) in Table 1 when $\gamma$ is close to $2 \omega_{m n}+\omega_{m \bar{n}}$ and assume

$$
\gamma=2 \omega_{m n}+\omega_{m \bar{n}}+\varepsilon \varsigma
$$

The terms $G_{1}$ and $G_{2}$ in the solvability equations (21) and (22) are modified to

$$
G_{1}=2 \alpha \bar{d}_{1} d_{2} \Lambda_{1} e^{i \varsigma T_{1}}
$$




$$
G_{2}=-\alpha d_{1}^{2} \Lambda_{1} e^{-i \varsigma T_{1}}
$$

It is noted that there always exists trivial solution $d_{1}=d_{2}=0$ in this case. To examine whether nontrivial solution exists, we follow the same procedure as before to obtain Eqs. (27), (28), (29), and (30) with $H_{1}, H_{2}, H_{3}$, and $H_{4}$ being modified to

$$
\begin{gathered}
H_{1}=-\frac{1}{2} \alpha a_{1} a_{2} \Lambda_{1} \sin \psi \\
H_{2}=4 \alpha a_{1} a_{2} \Lambda_{1} \cos \psi \\
H_{3}=-\frac{1}{4} \alpha a_{1}^{2} \Lambda_{1} \sin \psi \\
H_{4}=-2 \alpha a_{1}^{2} \Lambda_{1} \cos \psi
\end{gathered}
$$

where

$$
\psi=\varsigma T_{1}+\beta_{2}-2 \beta_{1}
$$

By the same procedure as before we can conclude that the nontrivial steady state solutions $a_{1}, a_{2}$, and $\psi$ must satisfy the following three equations,

$$
\begin{gathered}
c_{f} \omega_{m n}+\frac{1}{2} \alpha a_{2} \Lambda_{1} \sin \psi=0 \\
4 c_{f} \omega_{m \bar{n}} a_{2}+\alpha a_{1}^{2} \Lambda_{1} \sin \psi=0 \\
\alpha a_{2}\left(5 a_{2}^{2}+4 a_{1}^{2}+24 \Lambda_{1}^{2}+24 \Lambda_{2}^{2}\right)+2 \alpha \Lambda_{1}\left(a_{1}^{2}+4 a_{2}^{2}\right) \cos \psi \\
-8 \varsigma \hat{\omega}_{m n} a_{2}=0
\end{gathered}
$$

From Eqs. (49) and (50) we note that the steady state amplitudes $a_{1}$ and $a_{2}$ are related by

$$
\frac{a_{1}^{2}}{a_{2}^{2}}=\frac{2 \omega_{m \bar{n}}}{\omega_{m n}}
$$

The steady state vibration of the spinning disk is then

$$
\begin{aligned}
w(r, \theta, t)= & R_{m n}(r)\left[a_{1} \cos \left(\omega_{m n} t+\beta_{1}(t)+n \theta\right)\right. \\
& +a_{2} \cos \left(-\omega_{m \bar{n}} t+\beta_{2}(t)+n \theta\right) \\
& \left.+2 \Lambda_{1} \cos (\gamma t+n \theta)+2 \Lambda_{2} \cos (-\gamma t+n \theta)\right]+O(\varepsilon)
\end{aligned}
$$

It is noted that the frequencies of the free oscillation components in Eq. (53) are not exactly $\omega_{m n}$ and $\omega_{m \bar{n}}$. Instead, the frequencies are shifted by small amounts $\Delta \omega_{m n}$ and $\Delta \omega_{m \bar{n}}$, respectively, where

$$
\begin{aligned}
& \Delta \omega_{m n}=\frac{\varepsilon}{8 \hat{\omega}_{m n}}\left[\alpha\left(a_{1}^{2}+2 a_{2}^{2}+8 \Lambda_{1}^{2}+8 \Lambda_{2}^{2}\right)+4 \alpha a_{2} \Lambda_{1} \cos \psi\right] \\
& \Delta \omega_{m \bar{n}}=\frac{-\varepsilon}{8 \hat{\omega}_{m n}}\left[\alpha\left(a_{2}^{2}+2 a_{1}^{2}+8 \Lambda_{1}^{2}+8 \Lambda_{2}^{2}\right)+2 \alpha \frac{a_{1}^{2}}{a_{2}} \Lambda_{1} \cos \psi\right]
\end{aligned}
$$

Consequently, the final response $w(r, \theta, t)$ in Eq. (53) is quasiperiodic.

Figure 2 shows the steady state amplitudes $a_{1}, a_{2}$, and phase $\psi$ of a pair of $(0,3)$ modes as functions of $s$. The parameters used in the calculation are $\varepsilon=0.001, \quad \omega_{03}=11.94, \quad \omega_{03}=25.74, \quad q_{03}$ $=10000, c_{f}=0.2$, and $\alpha=0.4$. The solid and dashed lines represent the stable and unstable solutions, respectively. It is observed that secondary resonance is possible when frequency detuning parameter $s$ is greater than 3.63. The bifurcation at this critical point is of the saddle-node type. To examine the accuracy of the approximate solutions predicted by the multiple scale method, we use Runge-Kutta method to integrate Eq. (11) at $\varsigma=8$ with initial conditions $c_{03}(0)=9.98$ and $\dot{c}_{03}(0)=121.57 i$. The solid line in Fig. 3(a) shows the response history of the real part of $c_{03}$ after a long period of time. The dashed line represents the prediction
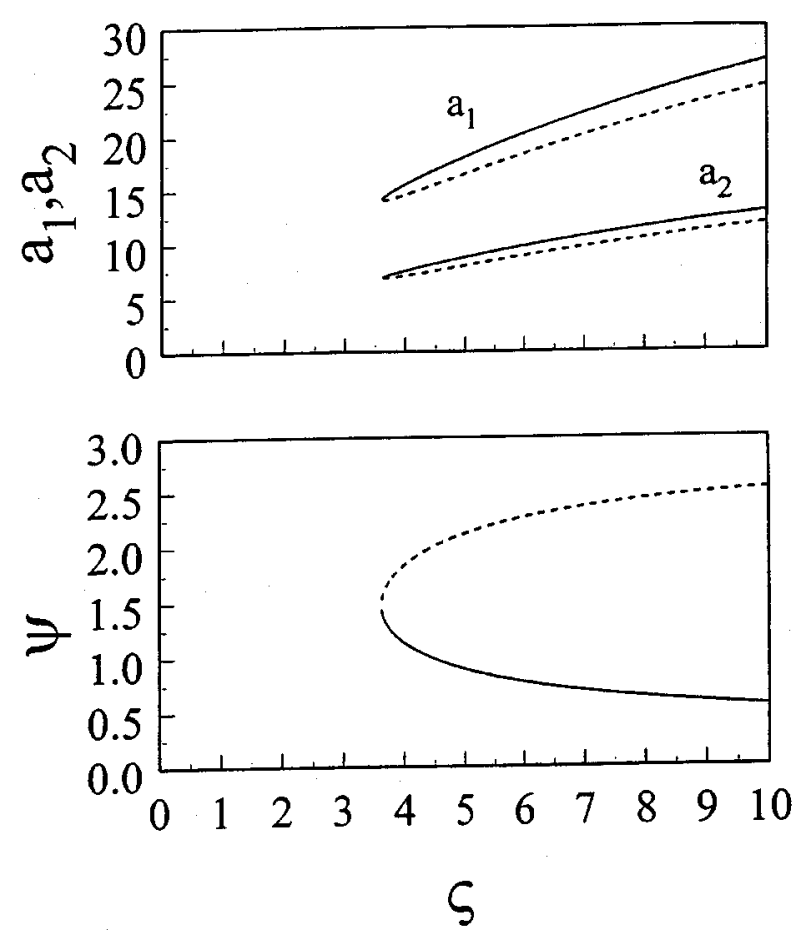

Fig. 2 Steady state solutions as functions of s when $\gamma=\mathbf{2} \omega_{03}$ $+\omega_{03}+\varepsilon s$, where $\omega_{03}=11.94, \omega_{03}=25.74$, and $\varepsilon=0.001$. Other parameters are $\Omega=2.3, \alpha=0.4, q_{03}=10,000$, and $c_{f}=0.2$.

from multiple scale method. Satisfactory accuracy is ensured. Figure 3(b) shows the Poincare map of the Runge Kutta solution recorded from $t=35000$ to 40000 with the sampling rate equal to the excitation frequency $\gamma$. The sampling points form a closed curve, which implies the quasiperiodic feature of the response. The analysis and the result for case (4) $\gamma \approx \omega_{m n}+2 \omega_{m \bar{n}}$ are similar to case (3).

For combination resonance case (5) in Table 1, we assume that

$$
\gamma=\frac{1}{2}\left(\omega_{m n}+\omega_{m \bar{n}}\right)+\varepsilon \varsigma
$$

The terms $G_{1}$, and $G_{2}$ in the solvability equations (21) and (22), and the terms $H_{1}, H_{2}, H_{3}$, and $H_{4}$ in Eqs. (27) to (30) are modified to

$$
\begin{gathered}
G_{1}=2 \alpha d_{2} \Lambda_{1} \Lambda_{2} e^{2 i s T_{1}} \\
G_{2}=-2 \alpha d_{1} \Lambda_{1} \Lambda_{2} e^{-2 i s T_{1}} \\
H_{1}=-\alpha a_{2} \Lambda_{1} \Lambda_{2} \sin \psi \\
H_{2}=8 \alpha a_{2} \Lambda_{1} \Lambda_{2} \cos \psi \\
H_{3}=-\alpha a_{1} \Lambda_{1} \Lambda_{2} \sin \psi \\
H_{4}=-8 \alpha a_{1} \Lambda_{1} \Lambda_{2} \cos \psi
\end{gathered}
$$

where

$$
\psi=2 \mathrm{~s}_{1} T_{1}+\beta_{2}-\beta_{1}
$$

As a consequence, the nontrivial steady state solutions $a_{1}, a_{2}$, and $\psi$ satisfy the following three equations

$$
\begin{gathered}
c_{f} \omega_{m n} a_{1}+\alpha a_{2} \Lambda_{1} \Lambda_{2} \sin \psi=0 \\
c_{f} \omega_{m \bar{n}} a_{2}+\alpha a_{1} \Lambda_{1} \Lambda_{2} \sin \psi=0 \\
\alpha a_{1} a_{2}\left[3 a_{2}^{2}+3 a_{1}^{2}+16\left(\Lambda_{1}^{2}+\Lambda_{2}^{2}\right)\right]+8 \alpha \Lambda_{1} \Lambda_{2}\left(a_{1}^{2}+a_{2}^{2}\right) \cos \psi \\
-16 s \hat{\omega}_{m n} a_{1} a_{2}=0
\end{gathered}
$$




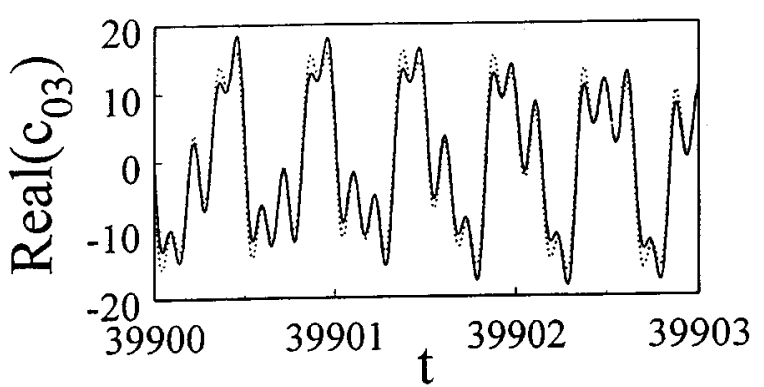

(a)

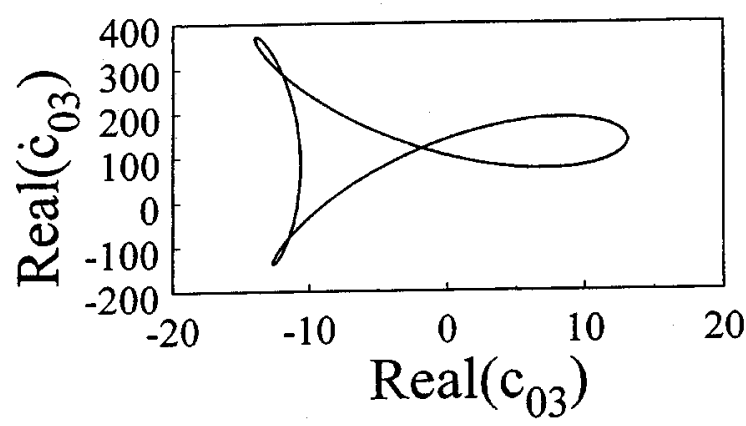

(b)

Fig. 3 (a) Time history and (b) the corresponding Poincare map when $s=8$ for the combination resonance case in Fig. 2. The initial conditions are $c_{03}(0)=9.98$ and $\dot{c}_{03}(0)=121.57 i$.

From Eqs. (64) and (65) we note that the steady state amplitudes $a_{1}$ and $a_{2}$ are related by

$$
\frac{a_{1}^{2}}{a_{2}^{2}}=\frac{\omega_{m \bar{n}}}{\omega_{m n}}
$$

The phase $\psi$ is independent of the excitation frequency,

$$
\psi=-\sin ^{-1}\left[\frac{c_{f} \sqrt{\kappa_{m n}}}{\alpha \Lambda_{1} \Lambda_{2}}\right]
$$

The final response $w(r, \theta, t)$ is in the same form as in Eq. (53).

Figure 4 shows the steady state amplitudes $a_{1}, a_{2}$, and phase $\psi$ as functions of $\mathrm{s}$. The parameters used in the calculation are $\varepsilon=0.001, \quad \omega_{03}=11.94, \quad \omega_{03}=25.74, \quad q_{03}=3000, \quad c_{f}=0.2, \quad$ and $\alpha=0.4$. It is observed that pitch-fork bifurcations occur at $s=0.805$ and 2.324. After studying the behaviors of the eigenvalues of the trivial solutions one can predict these two bifurcation frequencies analytically as

$$
\boldsymbol{\varsigma}_{c}=\frac{\alpha\left(\Lambda_{1}^{2}+\Lambda_{2}^{2}\right)}{\hat{\omega}_{m n}} \pm \sqrt{\frac{\alpha^{2} \Lambda_{1}^{2} \Lambda_{2}^{2}}{\kappa_{m n}}-c_{f}^{2}}
$$

From Eq. (69) we can also predict that the combination resonance of the summed type can occur only when the excitation amplitude $q_{m n}$ satisfies the following condition,

$$
q_{m n}^{2} \alpha \geqslant 4 c_{f} \sqrt{\kappa_{m n}}\left[4 n^{2} \gamma^{2} \Omega^{2}-\left(\kappa_{m n}-\gamma^{2}\right)^{2}\right]
$$

For the final case (6) in Table 1, we assume that

$$
\gamma=\frac{1}{2}\left(\omega_{m n}-\omega_{m \bar{n}}\right)+\varepsilon \varsigma
$$

The terms $G_{1}, G_{2}, H_{1}, H_{2}, H_{3}$, and $H_{4}$ are modified to

$$
\begin{gathered}
G_{1}=\alpha \bar{d}_{2} \Lambda_{2}^{2} e^{-2 i s T_{1}} \\
G_{2}=-\alpha \bar{d}_{1} \Lambda_{2}^{2} e^{-2 i s T_{1}}
\end{gathered}
$$
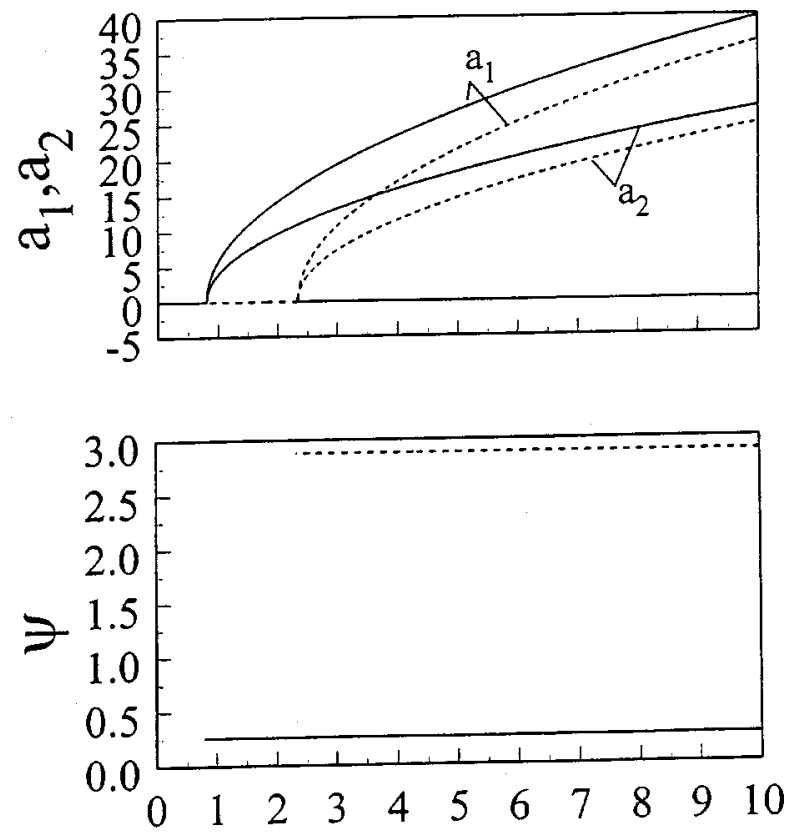

G

Fig. 4 Steady state solutions as functions of $s$ when $\gamma$ $=1 / 2\left(\omega_{03}+\omega_{03}\right)+\varepsilon s$, where $\omega_{03}=11.94, \quad \omega_{03}=25.74$, and $\varepsilon=0.001$. Other parameters are $\Omega=2.3, \alpha=0.4, q_{03}=3000$, and $c_{f}=0.2$.

$$
\begin{gathered}
H_{1}=\frac{1}{2} \alpha a_{2} \Lambda_{2}^{2} \sin \psi \\
H_{2}=4 \alpha a_{2} \Lambda_{2}^{2} \cos \psi \\
H_{3}=-\frac{1}{2} \alpha a_{1} \Lambda_{2}^{2} \sin \psi \\
H_{4}=-4 \alpha a_{1} \Lambda_{2}^{2} \cos \psi \\
\psi=2 \varsigma T_{1}+\beta_{2}+\beta_{1}
\end{gathered}
$$

As a consequence, the nontrivial steady state solutions $a_{1}, a_{2}$, and $\psi$ satisfy the following three equations

$$
\begin{gathered}
c_{f} \omega_{m n} a_{1}-\frac{1}{2} \alpha a_{2} \Lambda_{2}^{2} \sin \psi=0 \\
c_{f} \omega_{m \bar{n}} a_{2}+\frac{1}{2} \alpha a_{1} \Lambda_{2}^{2} \sin \psi=0 \\
\alpha a_{1} a_{2}\left(a_{2}^{2}-a_{1}^{2}\right)+4 \alpha \Lambda_{2}^{2}\left(a_{2}^{2}-a_{1}^{2}\right) \cos \psi+16 s \hat{\omega}_{m n} a_{1} a_{2}=0
\end{gathered}
$$

From Eqs. (79) and (80) we note that the steady state amplitudes $a_{1}$ and $a_{2}$ are related by

$$
a_{1}^{2} \omega_{m n}=-a_{2}^{2} \omega_{m \bar{n}}
$$

Therefore, the combination resonance of the difference type is impossible.

\section{Simultaneous Resonance}

Simultaneous resonance occurs when any two of the six closeness conditions in Table 1 are met at the same time. A careful examination of all possible combinations reveals that simulta- 

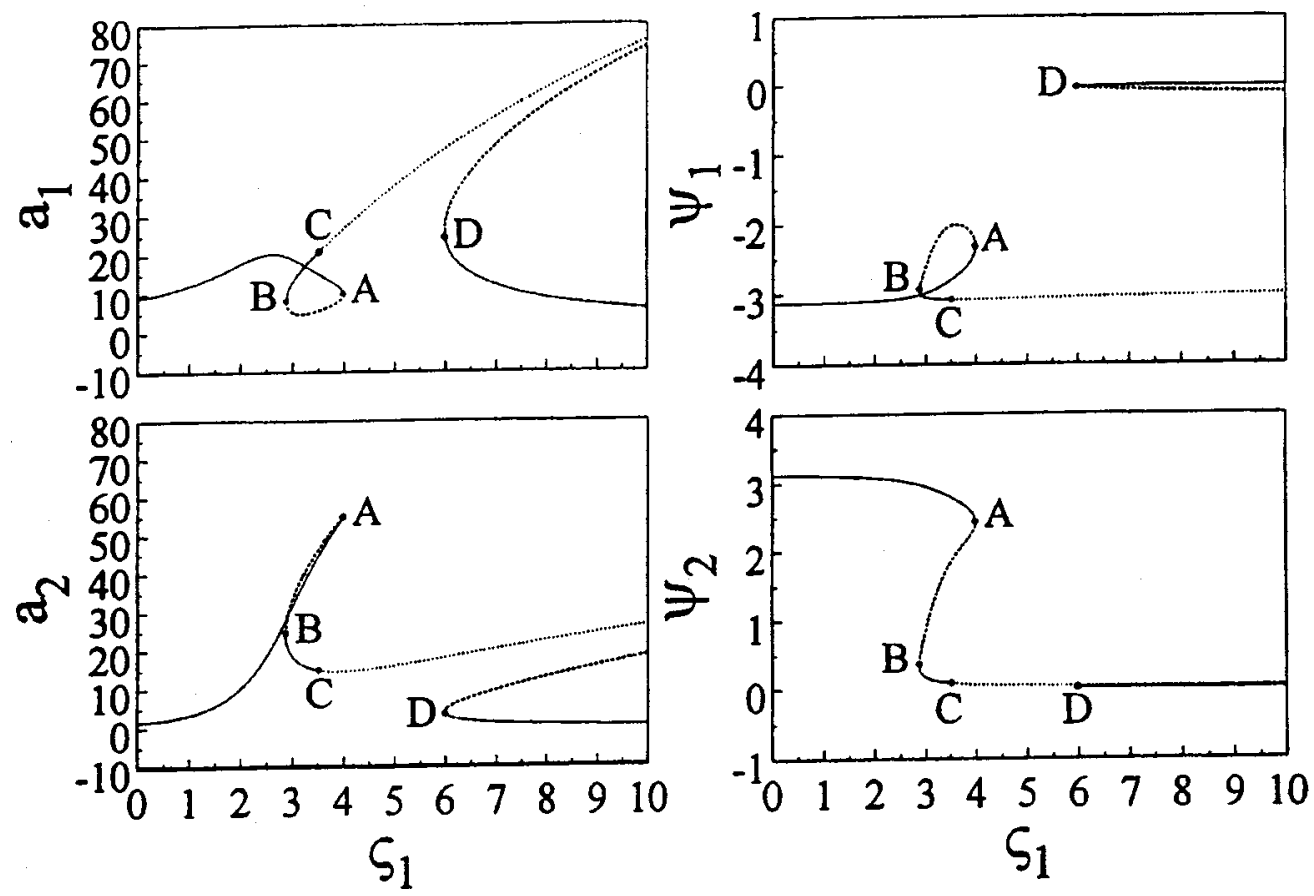

Fig. 5 Steady state solutions as functions of $s_{1}$ when $\gamma=1 / 3 \omega_{03}+\varepsilon \varsigma_{1}, \omega_{03}=14.13, \omega_{03}=23.55$, $\varepsilon=0.001$. Other parameters are $\Omega=1.57, \alpha=0.4, q_{03}=10,000$, and $c_{f}=0.2$.

neous resonance is possible only when cases (2) and (6) occur simultaneously. In other words, $\omega_{m \bar{n}}$ is close to $\frac{5}{3} \omega_{m n}$ and $\gamma$ is close to $\frac{1}{3} \omega_{m n}$. In this case we assume that

$$
\begin{gathered}
\gamma=\frac{1}{3} \omega_{m n}+\varepsilon \varsigma_{1} \\
\omega_{m \bar{n}}=\frac{5}{3} \omega_{m n}+\varepsilon \varsigma_{2}
\end{gathered}
$$

where $s_{1}$ and $s_{2}$ are two independent detuning parameters. The terms $G_{1}, G_{2}, H_{1}, H_{2}, H_{3}$, and $H_{4}$ are modified to

$$
\begin{gathered}
G_{1}=\alpha \Lambda_{1}^{2} \Lambda_{2} e^{i 3 s_{1} T_{1}}+\alpha \bar{d}_{2} \Lambda_{2}^{2} e^{i\left(s_{2}-2 \varsigma_{1}\right) T_{1}}=0 \\
G_{2}=\alpha \bar{d}_{1} \Lambda_{2}^{2} e^{i\left(\varsigma_{2}-2 \varsigma_{1}\right) T_{1}}=0 \\
H_{1}=-\alpha \Lambda_{1}^{2} \Lambda_{2} \sin \psi_{1}-\frac{1}{2} \alpha a_{2} \Lambda_{2}^{2} \sin \psi_{2} \\
H_{2}=8 \alpha \Lambda_{1}^{2} \Lambda_{2} \cos \psi_{1}+4 \alpha a_{2} \Lambda_{2}^{2} \cos \psi_{2} \\
H_{3}=\frac{1}{2} \alpha a_{1} \Lambda_{2}^{2} \sin \psi_{2} \\
H_{4}=-4 \alpha a_{1} \Lambda_{2}^{2} \cos \psi_{2}
\end{gathered}
$$

where

$$
\begin{gathered}
\psi_{1}=3 \varsigma_{1} T_{1}-\beta_{1} \\
\psi_{2}=\left(\varsigma_{2}-2 \varsigma_{1}\right) T_{1}-\beta_{1}-\beta_{2}
\end{gathered}
$$

Following a similar procedure we can conclude that the nontrivial steady state solutions of $a_{1}, \beta_{1}, a_{2}$, and $\beta_{2}$ must satisfy the following four equations,

$$
\begin{aligned}
& 24 \hat{\omega}_{m n} \varsigma_{1} a_{1}-\alpha a_{1}\left(a_{1}^{2}+2 a_{2}^{2}+8 \Lambda_{1}^{2}+8 \Lambda_{2}^{2}\right)-8 \alpha \Lambda_{1}^{2} \Lambda_{2} \cos \psi_{1} \\
& -4 \alpha a_{2} \Lambda_{2}^{2} \cos \psi_{2}=0 \\
& 2 c_{f} \omega_{m n} a_{1}+2 \alpha \Lambda_{1}^{2} \Lambda_{2} \sin \psi_{1}+\alpha a_{2} \Lambda_{2}^{2} \sin \psi_{2}=0
\end{aligned}
$$

$$
\begin{gathered}
8 \hat{\omega}_{m n}\left(\varsigma_{2}-5 \varsigma_{1}\right) a_{2}+\alpha a_{2}\left(a_{2}^{2}+2 a_{1}^{2}+8 \Lambda_{1}^{2}+8 \Lambda_{2}^{2}\right) \\
+4 \alpha a_{1} \Lambda_{2}^{2} \cos \psi_{2}=0 \\
2 c_{f} \omega_{m \bar{n}} a_{2}-\alpha a_{1} \Lambda_{2}^{2} \sin \psi_{2}=0
\end{gathered}
$$

It is noted that both $a_{1}$ and $a_{2}$ have to be nonzero if a nontrivial solution exists in Eqs. (93) through (96). In other words, singlemode resonance is not possible in this case. A straightforward solution procedure to solve Eqs. (93) to (96) for solutions $a_{1}, a_{2}$, $\psi_{1}$, and $\psi_{2}$ is described briefly in Appendix. The steady state vibration of the spinning disk is then

$$
\begin{aligned}
w(r, \theta, t)= & R_{m n}(r)\left[a_{1} \cos \left(3 \gamma t-\psi_{1}+n \theta\right)\right. \\
& +a_{2} \cos \left(-5 \gamma t+\psi_{1}-\psi_{2}+n \theta\right)+2 \Lambda_{1} \cos (\gamma t+n \theta) \\
& \left.+2 \Lambda_{2} \cos (-\gamma t+n \theta)\right]+O(\varepsilon)
\end{aligned}
$$

It is noted that the first term with frequency $3 \gamma$ on the right hand side of Eq. (97) is a backward mode excited directly by the external force in a superharmonic manner. The second term with frequency $5 \gamma$ is a forward mode excited internally by the vibration of the backward mode.

Figure 5 shows the amplitudes and phases of the steady state solutions as functions of detuning parameter $s_{1}$ for the internal resonance between a pair of $(0,3)$ modes at $\Omega=1.57$. The parameters used in the calculation are $\varepsilon=0.001, \omega_{03}=14.13, \omega_{03}$ $=23.55, q_{03}=10,000, c_{f}=0.2$, and $\alpha=0.4$. The excitation frequency $\gamma=1 / 3 \omega_{03}+\varepsilon \varsigma_{1}$. The stable solution branch from $\varsigma_{1}=0$ undergoes a saddle-node bifurcation at $\varsigma_{1}=3.99$ (point A), creating an unstable branch BA. At point B the unstable branch undergoes another saddle-node bifurcation creating a stable branch BC. The branch $\mathrm{BC}$ then undergoes a super-critical Hopf bifurcation at point $\mathrm{C}$, creating a quasiperiodic solution $c_{03}$, which cannot be shown in Fig. 5. Point D is a saddle-node bifurcation point for additional branch of solutions.

To demonstrate the existence of Hopf bifurcation predicted by the multiple scale analysis we use Runge-Kutta method to integrate Eq. (11) at $\varsigma_{1}=7$ with initial conditions $c_{03}=-4.96$ 


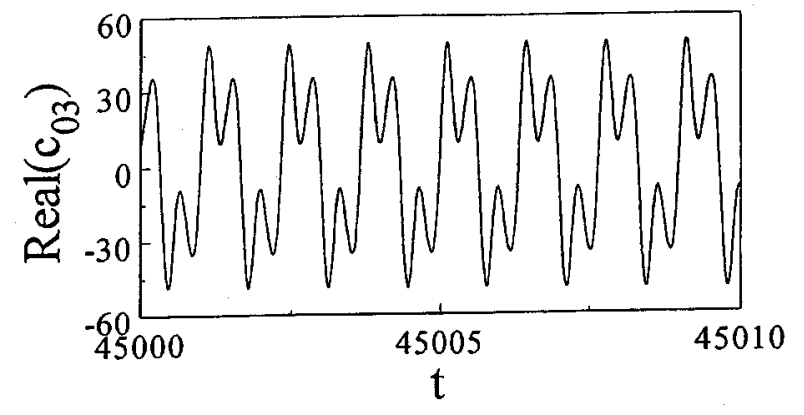

(a)

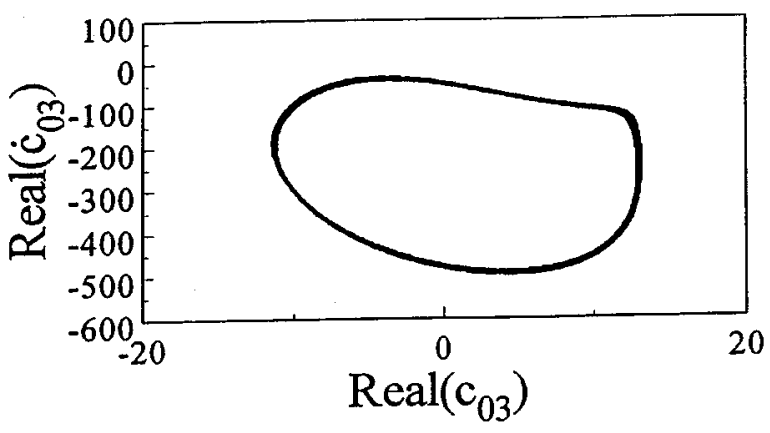

(b)

Fig. 6 (a) Time history and (b) the corresponding Poincare map when $s_{1}=7$ for the simultaneous resonance case in Fig. 5. The initial conditions are $c_{03}(0)=-4.96+i 2.15$ and $\dot{c}_{03}(0)$ $=-130.26+i 50.83$.

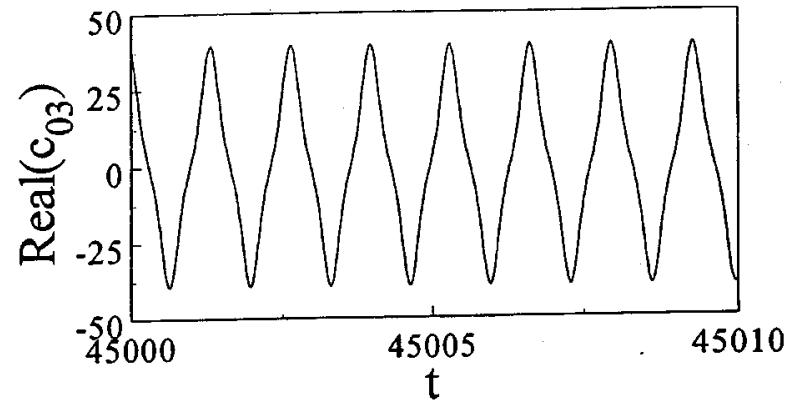

(a)

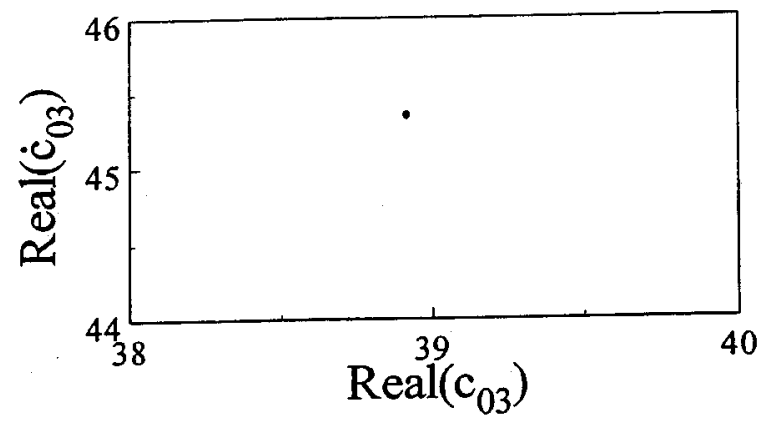

(b)

Fig. 7 (a) Time history and (b) the corresponding Poincare map when $s_{1}=7$ for the simultaneous resonance case in Fig. 5. The initial conditions are $c_{03}(0)=0$ and $\dot{c}_{03}(0)=0$.
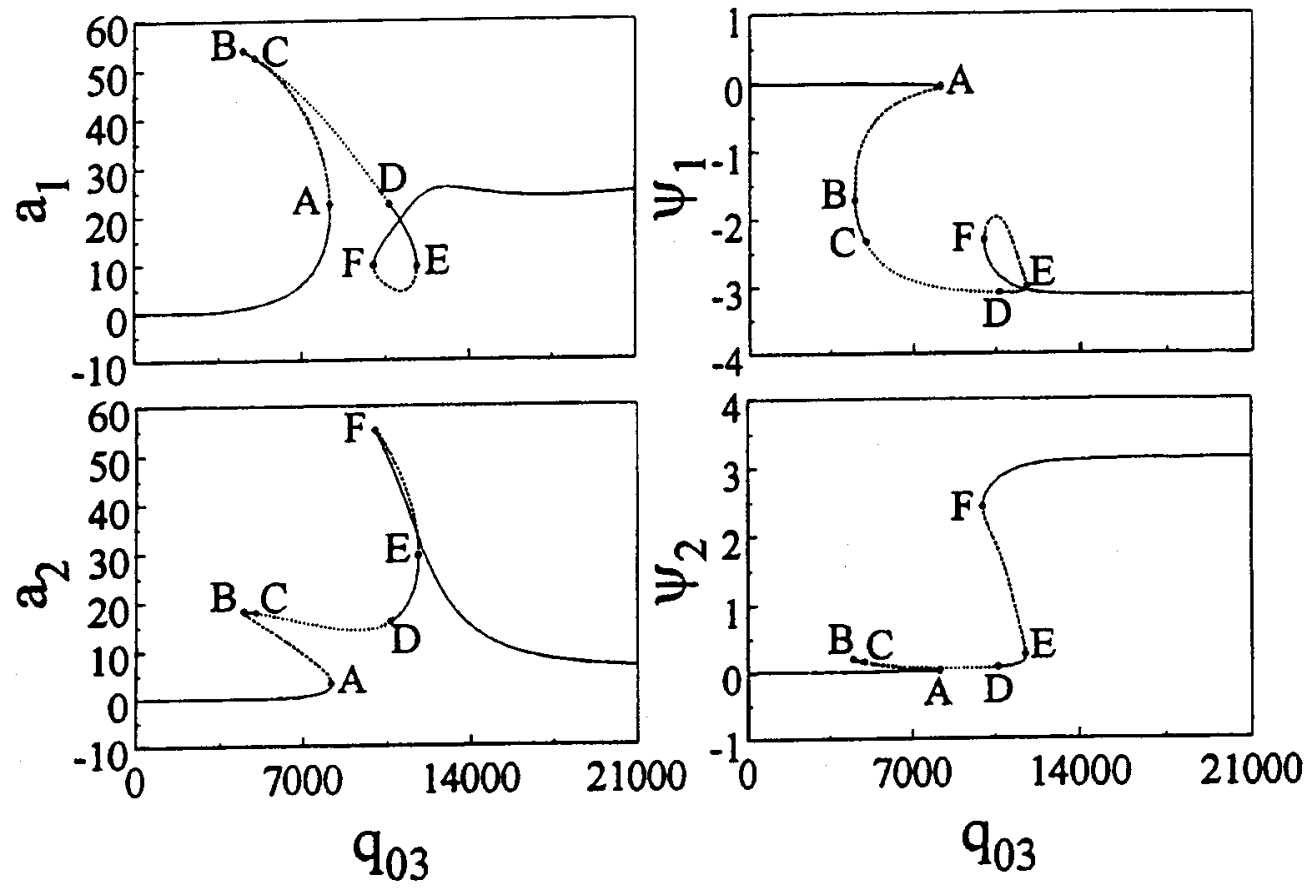

Fig. 8 Steady state solutions of simultaneous resonance as functions of loading parameter $q_{03}$ when $\mathrm{s}_{1}=4$ 
$+i 2.15$ and $\dot{c}_{03}=-130.26+i 50.83$. Figure 6(a) shows the response history of the real part of $c_{03}$ after a long period of time. Figure 6(b) shows its Poincare map recorded from $t=45,000$ to 50,000 with the sampling rate equal to the excitation frequency. The sampling points fill up a small strip around a closed curve, which implies the quasiperiodic feature of the response induced by a Hopf bifurcation. If the initial conditions are changed to $c_{03}=0$ and $\dot{c}_{03}=0$, the response then settles to the stable branch to the right of point D in Fig. 5, as shown in Fig. 7. The Poincare map in Fig. 7(b) records the sampling points from $t=20,000$ to 25,000 , which indicates clearly that the response is periodic. It is noted that the amplitude of the quasiperiodic solution branch after point $\mathrm{C}$ grows with $s_{1}$. We also take note of the closing gap between the unstable branches following points $\mathrm{C}$ and $\mathrm{D}$. At certain point of $s_{1}$ the domain of attraction of the quasiperiodic solution touches the stable manifold of the saddle point in the neighborhood. Beyond this $s_{1}$ the quasiperiodic solution will no longer exist. We estimate numerically that it occurs around $s_{1}=19$.

Figure 8 shows the steady state solutions as functions of loading parameter $q_{03}$ when $\mathrm{s}_{1}=4$. Other parameters are the same as those used in Fig. 5. The stable branch undergoes saddle-node bifurcation at point $\mathrm{A}$ and another saddle-node bifurcation at $\mathrm{B}$. The stable branch starting from point $B$ then undergoes a supercritical Hopf bifurcation at point $\mathrm{C}$ creating an unstable periodic solution and a quasiperiodic solution. The unstable branch CD undergoes a super-critical Hopf bifurcation at point D. Points E and $\mathrm{F}$ are two saddle-node bifurcation points. It is noted that all the stable periodic solutions predicted in Figs. 5 and 8 can be realized by integrating Eq. (11) numerically.

It is interesting to note that when the closeness condition (6) in Table 1 is met alone, we predict that combination resonance of the difference type is impossible. However, when conditions (2) and (6) in Table 1 are met at the same time, complicated response is induced and both the backward and the forward modes are excited via internal resonance.

\section{Conclusions}

In this paper we investigate the possibility of secondary resonance of a spinning disk under space-fixed excitations. Von Karman's plate model is employed in formulating the equations of motion of the spinning disk. Galerkin's procedure is used to discretize the equations of motion. Attention is focused on the nonlinear coupling between a pair of forward (with frequency $\omega_{m \bar{n}}$ ) and backward (with frequency $\omega_{m n}$ ) traveling modes. Multiple scale method is used to predict the steady state periodic solutions. The secondary oscillations are classified into three types, i.e., single-mode resonance, combination resonance, and simultaneous resonance. The accuracy of steady state solution predicted by multiple scale method is examined by direct integration with RungeKutta method. Several conclusions can be summarized as follows.

(1) When the excitation frequency is close to $1 / 3 \omega_{m \bar{n}}$ or $1 / 3 \omega_{m n}$, then single-mode superharmonic resonance will be induced. The response behavior is similar to a classical Duffing oscillator.

(2) In the case when the excitation frequency is close to $2 \omega_{m n}+\omega_{m \bar{n}}$ or $\omega_{m n}+2 \omega_{m \bar{n}}$, both the forward and the backward modes will be excited when the frequency detuning parameter is greater than a critical value. When the combination resonance does occur, the frequencies of the free oscillation components are shifted slightly from the respective natural frequencies $\omega_{m \bar{n}}$ and $\omega_{m n}$. The final response is therefore quasiperiodic. The bifurcation points in the bifurcation diagram are of the saddle-node type.

(3) In the case when the excitation frequency is close to $1 / 2\left(\omega_{m \bar{n}}+\omega_{m n}\right)$, again both the forward and the backward modes will be excited. The bifurcation points in the bifurcation diagram are of the pitch-fork type.

(4) In the case when the excitation frequency is close to $1 / 2\left(\omega_{m \bar{n}}-\omega_{m n}\right)$, no combination resonance is possible.
(5) In the case when the excitation frequency is close to $1 / 3 \omega_{m n}$ and $1 / 2\left(\omega_{m \bar{n}}-\omega_{m n}\right)$ simultaneously, internal resonance between the forward and backward modes can occur. The frequencies of the free oscillation components are exactly 3 times and 5 times that of the excitation frequency. In this special case both saddle-node and Hopf bifurcations are observed.

\section{Acknowledgments}

The results presented here were obtained in the course of research supported by a grant from the National Science Council of the Republic of China.

\section{Appendix}

By eliminating $\psi_{2}$ from Eqs. (93) and (94) we can derive an equation involving only $g=a_{1}^{2}$ and $f=a_{2}^{2}$ in the form

$$
p_{2}(f) g^{2}+p_{1}(f) g+p_{0}(f)=0
$$

With use of Eqs. (93) and (94), Eqs. (91) and (92) can be reduced to

$$
q_{4}(f) g^{4}+q_{3}(f) g^{3}+q_{2}(f) g^{2}+q_{1}(f) g+q_{0}(f)=0
$$

$p_{i}$ and $q_{i}$ are functions of $f$ only. Equations $(A 1)$ and $(A 2)$ can then be reduced to the following equation in terms of $f$,

$$
\left(m_{2} p_{0}-m_{0} p_{2}\right)^{2}+\left(m_{2} p_{1}-m_{1} p_{2}\right)\left(m_{0} p_{1}-m_{1} p_{0}\right)=0
$$

where

$$
\begin{gathered}
m_{2}=p_{0}\left(p_{2} q_{3}-p_{1} q_{4}\right) \\
m_{1}=p_{0}\left(p_{2} q_{2}-p_{0} q_{4}\right)-p_{2}^{2} q_{0} \\
m_{0}=p_{0} p_{2} q_{1}-p_{1} p_{2} q_{0}
\end{gathered}
$$

After solving $a_{2}$ from Eq. (A3), $a_{1}$ can be calculated from Eq. $(A 1)$.

\section{References}

[1] Benson, R. C., and Bogy, D. B., 1978, "Deflection of a Very Flexible Spinning Disk Due to a Stationary Transverse Load," ASME J. Appl. Mech., 45, pp. 636-642.

[2] Cole, K. A., and Benson, R. C., 1988, "Fast Eigenfunction Approach for Computing Spinning Disk Deflections,” ASME J. Appl. Mech., 55, pp. 453457.

[3] Ono, K., and Maeno, T., 1987, "Theoretical and Experimental Investigation on Dynamic Characteristics of a 3.5-Inch Flexible Disk Due to a Point Contact Head," Tribology and Mechanics of Magnetic Storage Systems, 3, SP.21, (STLE), pp. 144-151.

[4] Jiang, Z. W., Chonan, S., and Abe, H., 1990, "Dynamic Response of a Read/ Write Head Floppy Disk System Subjected to Axial Excitation," ASME J. Vibr. Acoust., 112, pp. 53-58.

[5] Chen, J. S., and Hsu, C. M., 1997, "On the Transient Response of a Spinning Disk Under a Space-Fixed Step Load,” ASME J. Appl. Mech., 64, pp. 10171019

[6] Chen, J. S., and Hsu, C. M., 1997, "Forced Response of a Spinning Disk Under Space-Fixed Couples," J. Sound Vib., 206, pp. 627-639.

[7] Nowinski, J. L., 1964, "Nonlinear Transverse Vibrations of a Spinning Disk," ASME J. Appl. Mech., 31, pp. 72-78

[8] Tobias, S. A., and Arnold, R. N., 1957, "The Influence of Dynamical Imperfection on the Vibration of Rotating Disks," Proc. Inst. Mech. Eng., 171, pp. 669-690.

[9] Maher, J. F., and Adams, G. G., 1991, "The Point-Load Solution Using Linearized von Karman Plate Theory for a Spinning Flexible Disk Near a Baseplate," STLE/ASME Tribology Conference, St. Louis, Missouri, pp. 1-9.

[10] Torii, T., Yasuda, K., and Toyada, T., 1998, "Nonlinear Forced Oscillation of a Rotating Disk Excited at a Point Fixed in Space," JSME Int. J., Ser. C, 41, pp. 592-598.

[11] Raman, A., and Mote, Jr., C. D., 1999, "Non-linear Oscillations of Circular Plates Near a Critical Speed Resonance," Int. J. Non-Linear Mech., 34, pp. 139-157.

[12] Chen, J. S., 1999, "Steady State Deflection of a Circular Plate Rotating Near Its Critical Speed,” ASME J. Appl. Mech., 66, pp. 1015-1017.

[13] Nayfeh, T. A., and Vakakis, A. F., 1994, "Subharmonic Travelling Waves in a Geometrically Non-Linear Circular Plate," Int. J. Non-Linear Mech., 29, pp. 233-245.

[14] Nayfeh, A. H., and Mook, D. T., 1979, Nonlinear Oscillations, Wiley, New York. 